\title{
Diabetic peripheral neuropathy: when patients present with pain
}

\section{Introduction}

Diabetes mellitus (DM) is a chronic medical condition affecting 29million Americans and over 360million people world-wide. ${ }^{1}$ Diabetic polyneuropathy (DPN) is a common, debilitating, and distressing microvascular complication of diabetes that develops in up to $50 \%$ of patients with DM. ${ }^{2}$ This disorder is defined as the presence of symptoms and/or signs of peripheral nerve dysfunction in patients with diabetes after exclusion of all other causes of neuropathy. ${ }^{3}$ Neuropathy is usually a late finding in type 1 diabetes; however, it can be an early finding in type 2 diabetes - sometimes present at the time of diagnosis. DPN, also referred to as distal symmetrical sensory polyneuropathy (DSP), has two main clinical consequences: diabetic peripheral neuropathic pain (DPNP) and the insensate foot which predisposes to neuropathic foot ulceration. The prevalence of DPNP was $34 \%$ in a 2011 UK study. ${ }^{4}$ Extrapolating, this would be about 10 million in the US and over 120million people worldwide.

Risk factors for DPN include both modifiable and non-modifiable. Non-modifiable risk factors include: older age, longer duration of diabetes, genetic profile, height and gender (males $>$ females). Modifiable risk factors include: hypertension, hyperglycemia (DM \& pre-DM), dyslipidemia, smoking, B6 and B12 deficiency, metformin usage, obesity, peripheral arterial disease and heavy alcohol use. $^{5}$ Diabetes mellitus is the most common cause of peripheral polyneuropathy but it is important to rule out other treatable coexisting disorders (e.g. hypothyroidism, celiac sprue, uremia) or contributing factors (e.g. alcohol abuse, B12 deficiency and metformin usage). In one study, $55 \%$ of patients with DPN had an additional potential cause of polyneuropathy. ${ }^{6}$ A good medical history and physical exam including a focused neurologic exam will make the diagnosis of DPN in most cases. DPN is typically a symmetrical distal and primarily a sensory neuropathy. In atypical cases, additional testing (e.g. electrophysiologic testing and CT or an MRI of the spine) is required to exclude other conditions. ${ }^{5}$

\section{Diabetic Peripheral Neuropathic Pain (DPNP)}

In 1931, Dr. Charles Mayo stated that "of all the symptoms for which physicians are consulted, pain in one form or the other, is the most common and most urgent." Diabetic peripheral neuropathic pain (DPNP) is chronic, insidious, and symmetrical and primarily affects the lower extremities. Typically, the pain begins distally in the feet and then moves proximally ("stocking and glove" distribution). Common locations of pain include the toes, the metatarsal areas and dorsum of the feet, heels and calves. Advanced, DPN may involve the fingers and hands.

DPNP may present with three distinct types of pain: dysesthesic, paresthetic and muscular. Dysesthesic pain presents as a burning whereas paresthetic pain typically presents as pins and needles sensation. Both of these pains are mediated through small fibers. In contradistinction, large fiber neuropathy more commonly presents as a deep, dull, or aching muscular pain. In many patients there is a mixed neuropathy. The pain and paresthesias are typically worse at
Volume 3 Issue 2 - 2016

\author{
Robert Tanenberg \\ Department of Endocrinology, East Carolina University, USA
}

Correspondence: Robert Tanenberg, Department of Endocrinology, East Carolina University, USA, Tel 2527442567 . EmailTANENBERGR@ecu.edu

Received: January 18, 2016 | Published: February 26, 2016

night and when patients are tired or stressed. ${ }^{8}$ Pain, often accompanied by sensory loss, may persist over several years leading to significant disability in some patients. ${ }^{8}$

\section{Pharmacologic options for DPNP}

Physicians currently have several good therapeutic medications for controlling DPNP. However, none of these agents have been demonstrated to modify the disease state. Smoking cessation is essential for pain control and must be addressed. ${ }^{5}$ According to an expert consensus, only the drug pregabalin has "Level A" evidence for pain control in DPNP. Agents with "Level B" evidence include duloxetine, gabapentin, amitriptyline and tramadol. ${ }^{9}$ Recently, our group demonstrated that duloxetine was superior to pregabalin in a select subgroup of patients not on concomitant antidepressants. ${ }^{10}$ Therapeutic response to combination therapy with agents from two classes have had mixed results. ${ }^{11,12}$ An excellent algorithm for the treatment of DPNP is reproduced in Figure $1 .{ }^{13}$

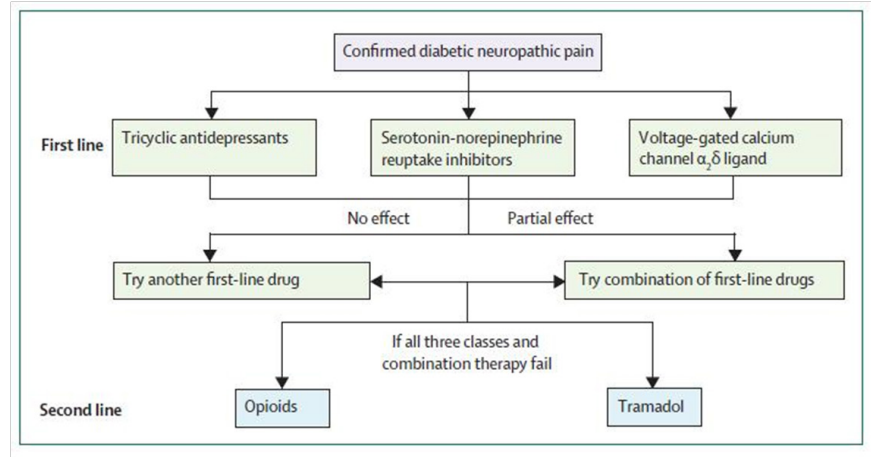

Figure I Treatment Algorithm for Diabetic Neuropathic Pain

\section{Conclusion}

Diabetic Peripheral Polyneuropathy is a very common microvascular complication affecting millions of people with both types of diabetes. About $1 / 3$ of patients present with PDPN and most will have their pain controlled but not eliminated with agents that do change the underlying pathologic mechanisms. Early and aggressive control of glucose, blood pressure and lipids are essential to improve the disorder. Further research is needed for more effective agents with better side effect profiles. 


\section{Acknowledgements}

None.

\section{Conflict of interest}

Author declares that there is no conflict of interest.

\section{References}

1. CDC. 2014 National Diabetes Statistics Report. Centers for disease controls and prevention. 2014.

2. Shaw JE, Zimmet PZ. The epidemiology of diabetic neuropathy. Diabetes Rev. 1999;7:245-252.

3. Boulton AJ, Gries FA, Jervell JA. Guidelines for the diagnosis and outpatient management of diabetic peripheral neuropathy. Diabet Med. 1998;15(6):508-514.

4. Abbott CA, Malik RA, Van Ross ER, et al. Prevalence and characteristics of painful diabetic neuropathy in a large community-based diabetic population in the U.K. Diabetes Care. 2011;34(10):2220-2224.

5. Tanenberg RJ, Donofrio PD. Neuropathic Problems of the Lower Extremities in Diabetic Patients. In: Levin, O'Neal's, editors. The Diabetic Foot 7th ed. In: Pfeifer MA, Bowker J, editors. Philadelphia: Mosby Elsevier; 2008;33-74p.

6. Gorson KC, Ropper AH. Additional causes for distal sensory polyneuropathy in diabetic patients. J Neurol Neurosurg Psychiatry. 2007;77(3):354-358.
7. Mayo clinic. Mayo Brothers. Sharing Mayo Clinic. 2016.

8. Galer BS, Giana A, Jensen MP. Painful diabetic polyneuropathy: epidemiology, pain description, and quality of life. Diabetes Res Clin Pract. 2000;47(2):123-128.

9. Bril V, England J, Franklin GM, et al. Evidence-based guideline: treatment of painful neuropathy. Report of the American Academy of Neurology, the American association of Neuromuscular and Electrodiagnostic Medicine, and the American Academy of Physical Medicine and Rehabilitation. Neurology. 2011;76(20):1758-1765.

10. Tanenberg RJ, Clemow DB, Giaconia JM, et al. Duloxetine Compared with Pregabalin for Diabetic Peripheral Neuropathic Pain Management in Patients with Suboptimal Pain Response to Gabapentin and Treated without Antidepressants: a Post Hoc Analysis. Pain Pract. 2014;14(7):640-648.

11. Iyer S, Tanenberg RJ. Pharmacologic Management of Diabetic Peripheral Neuropathic Pain. Expert Opin Pharmacother. 2013;14(13):1765-1775.

12. Tanenberg RJ, Irving G, Risser R, et al. An Open-Label, Randomized, Noninferiority Comparison of Duloxetine, Pregabalin, and the Combination of Duloxetine and Gabapentin for the Management of Diabetic Peripheral Neuropathic Pain in Patients with Inadequate Response to Gabapentin: an open-label, randomized, noninferiority comparison. Mayo Clinic Proceedings. 2011;86(7):615-626.

13. Callaghan BC, Cheng HT, Stables CL, et al. Diabetic neuropathy: clinical manifestations and current treatments. Lancet Neurol. 2012;11(6):521-534. 